\title{
Terapia del dolore: nuove norme e aspetti pratici per l'uso degli analgesici
}

\begin{abstract}
Until recently, pain therapy in Italy was conducted with largely sub-optimal standards, as revealed by one of the lowest mean morphine consumption values, an important indicator of pain therapy quality according to the WHO, among industrialized countries. The recognition of such a negative situation has led to an important effort to improvement, carried out by the law-maker, institutions and health professionals. As suggested by the WHO, these efforts aimed at three main objectives: reducing the cultural barriers to appropriate opioid use through targeted education and information, easing of analgesic drug prescription and availability, and improvement of the integration among hospital-based and domiciliar pain management and care-giving. In this article, the new legal framework concerning the prescription and administration of analgesics regulatory innovations started in February 2001 - is presented and its practical implications for health professionals are discussed. Alongside the new laws, some regulatory interventions recently adopted by the Italian Drug Agency (AIFA) are presented and discussed, as they share the proposition of simplifying pain therapy and permit the prescription of more analgesics paid for by the National Health Service.

In the last years, the premises for a more rational and modern approach to pain therapy in Italy have been created, which will need to be followed by cultural, organizing and clinical practice adaptations in order to warrant effective and efficient management of algic patients.
\end{abstract}

Keywords: pain therapy, legal aspects, opioid analgesics Farmeconomia e percorsi terapeutici 2005; 6 (1): 77-83

\section{INTRODUZIONE}

In Italia, fino a pochi anni fa, la terapia del dolore presentava alcuni limiti, in particolare per la mancanza di un approccio culturale corretto all'utilizzo degli analgesici oppioidi da parte della popolazione medica, con conseguenti ricadute nella pratica clinica, oltre che per gli ostacoli posti da una legislazione molto restrittiva. L'OMS considera il consumo pro capite di morfina un indice importante della qualità di un Sistema Sanitario Nazionale. La dose pro-capite media annuale di morfina consumata nel quinquennio 1993-1997 è stata di 73,9 mg per la Danimarca, di 28,8 per la Francia, 20,5 mg per l'Inghilterra e $2 \mathrm{mg}$ per l'Italia [1]. Partendo da questo dato negativo, negli ultimi anni professionisti, amministratori ed enti regolatori si sono impegnati in uno sforzo teso a migliorare la qualità dell'assistenza antalgica in Italia, sviluppando interventi in tre direzioni principali:

- facilitare la disponibilità e la prescrivibilità dei farmaci antalgici, in particolare degli oppioidi per utilizzo terapeutico;

- approntare forme organizzative che consentano una continuità di intervento;
- favorire la formazione degli operatori sanitari in questo settore e l'informazione divulgativa all'opinione pubblica.

\section{FORMAZIONE E INFORMAZIONE PER RIDURRE LE BARRIERE CULTURALI}

Gli ostacoli ad un corretto trattamento del dolore cronico sono stati identificati e categorizzati dall'OMS in barriere dovute a:

- paziente

- professionisti

- istituzioni [1].

L'approccio alla rimozione delle barriere dovute al paziente e ai familiari (riluttanza a riportare e descrivere il dolore, a seguire le prescrizioni, timore riguardo alla tolleranza, alla dipendenza e agli effetti collaterali, supina accettazione del dolore, timore di iniezioni ecc.) ha trovato realizzazione principalmente nella produzione di opuscoli informativi e nell'allestimento di attività culturali rivolte al pubblico, quali le "giornate di sollievo dal dolore". 
Le barriere dovute ai professionisti, in parte sovrapponibili a quelle dovute ai pazienti, sono anch'esse di tipo prevalentemente culturale, come l'incapacità a rilevare metodicamente e a trattare razionalmente il dolore, con limitate conoscenze scientifiche sulle proprietà farmacologiche degli oppioidi e la confusione concettuale fra tolleranza, dipendenza fisica e dipendenza psicologica dagli oppioidi [2].

La dipendenza psicologica è un fenomeno del tutto assente nel trattamento del dolore cronico: dati di letteratura attribuiscono un'incidenza dell'ordine di 4 casi su 12.000 pazienti trattati [3]. Un cenno merita la "pseudodipendenza". Si tratta di una sindrome creata dalla cattiva pratica clinica: i pazienti che continuano a fare esperienza di dolore non controllato o di ricomparsa in tempi brevi, manifestano preoccupazione riguardo alla disponibilità di farmaci oppioidi e comportamento di desiderio del farmaco tali da simulare una dipendenza psicologica, che cessa però una volta che il dolore sia ben controllato da un incremento di dose.

\section{FACILITARE LA DISPONIBILITÀ E LA PRESCRIVIBILITÀ DEI FARMACI ANTALGICI}

Il quadro normativo italiano precedente al 2001, logica conseguenza di questo contesto culturale, era rappresentato dal testo unico delle leggi in materia di disciplina degli stupefacenti e sostanze psicotrope (D.P.R.309/90) [4], orientato principalmente a normare in modo rigido la movimentazione delle sostanze stupefacenti, a reprimere le attività illecite e prevenire gli stati di tossicodipendenza, senza porre attenzione all'impiego terapeutico delle sostanze stupefacenti.

Una tale normativa, così penalizzante la prescrizione e la distribuzione degli oppioidi, nasce da un equivoco culturale purtroppo diffuso, cioè che a una maggiore disponibilità per uso terapeutico corrisponda una maggiore diffusione dell'utilizzo voluttuario.

Questa convinzione è stata smentita da numerosi lavori e revisioni nazionali e internazionali: ad esempio, il lavoro di Joranson [5] ha evidenziato una riduzione percentuale dell'abuso di oppioidi dell' $1,3 \%$ sul totale di abuso per qualunque farmaco, a fronte di un incremento percentuale nell'utilizzo terapeutico di oppioidi variabile, a seconda del singolo farmaco, da più 19\% a più $1168 \%$ nel periodo di tempo che va dal 1990 al 1996.

L'abuso di oppioidi andava, dunque, in senso esattamente opposto all'incremento dell'utilizzo terapeutico dei farmaci e, con il 3,8\% delle segnalazioni, l'abuso di oppioidi era agli ultimi posti della classifica di abuso dei farmaci, dopo analgesici non oppioidi $(8,6 \%)$, alcool associato a farmaci $(18,3 \%)$, farmaci illegali $(33,2 \%)$ e altri farmaci $(36,1 \%)$.

Coerentemente con le visioni più moderne, il Parlamento italiano ha approvato una serie di leggi per migliorare le possibilità terapeutiche dei pazienti algici. I riferimenti normativi attuali originano dalla Legge n. 12, 8 Febbraio 2001 : "Norme per agevolare l'impiego dei farmaci analgesici oppiacei nella terapia del dolore" (allegato 1) [6], integrata dai successivi: Decreto 24 maggio 2001 G.U. 133 del 11/6/2001 "Approvazione del ricettario per la prescrizione dei farmaci di cui all'allegato III bis del D.P.R. 9 ottobre $1990 n^{\circ} 309$ introdotto dalla Legge 8 febbraio 2001 n $^{\circ} 12$ " [7], Decreto 3 agosto 2001 G.U. n 204 del 3/9/2001 “Approvazione del registro di carico e scarico delle sostanze stupefacenti e psicotrope per le unità operative" [8] e Decreto 4 aprile 2003 G.U. n 122 del 28/ $5 / 2003$ "Modifiche ed integrazioni al Decreto del Ministro della Sanità del 24 maggio 2001" [9].

Le principali novità contenute in questa nuova legislazione tese a facilitare la prescrivibilità degli analgesici sono:

- individuazione di un elenco di farmaci (buprenorfina, codeina, diidrocodeina, fentanyl, idrocodone, idromorfone, metadone, morfina, ossicodone, ossimorfone) che godono di modalità prescrittive semplificate rispetto alla normativa precedente;

- estensione della validità delle ricette degli stupefacenti a trenta giorni, escluso quello di emissione;

- ricettazione su un ricettario, sempre personale, confezionato in blocchetti da trenta ricette numerate, autocopiante a ricalco, rilasciato dall' Azienda Sanitaria Locale, con valenza su tutto il territorio nazionale;

- la ricetta, redatta in triplice copia per la fornitura a carico del S.S.N. e in duplice copia per le cessioni al di fuori del SSN, deve essere compilata in ogni sua parte e corredata di timbro personale del medico prescrittore, contenente l'indicazione del 
suo domicilio e del numero di telefono professionale. La firma deve essere apposta dal medico in originale sulla prima pagina;

- non vi è più l'obbligo di indicare l'indirizzo di residenza dell'assistito;

- la prescrizione può essere compilata senza l'obbligo di utilizzare le "tutte lettere" per scrivere il dosaggio, il modo e il tempo di somministrazione, il numero di confezioni e la durata del trattamento e possono essere utilizzate le normali contrazioni;

- la ricetta può contenere fino a due preparazioni o due dosaggi per una cura di durata non superiore a trenta giorni. La posologia indicata deve comportare che l'assunzione dei medicinali prescritti sia completata entro trenta giorni;

- salvo i casi in cui sia necessario modificare la terapia, la prescrizione non può essere ripetuta prima del completamento della terapia indicata con la precedente prescrizione;

- anche i medicinali contenenti farmaci compresi nelle tabelle I, II e III e nell' Allegato III-bis, in associazione con altri farmaci e collocati nella tabella $\mathrm{V}$, se usati nella terapia del dolore severo in corso di patologia neoplastica o degenerativa a carico del SSN, devono essere prescritti su ricettario a ricalco, per una terapia non superiore a trenta giorni; per le altre indicazioni questi farmaci, tuttavia, continuano a poter essere prescritti con la semplice ricetta non ripetibile (RNR);

- in caso di prescrizione su ricetta a ricalco da parte di specialista di struttura sanitaria convenzionata, i medicinali possono essere dispensati dalle farmacie in regime di convenzione. Nello spazio della ricetta destinato all'indirizzo professionale del medico, deve essere riportata la denominazione e l'indirizzo della struttura sanitaria convenzionata con il S.S.N. in cui svolge l'attività il medico;

- nel caso in cui la ricetta a ricalco sia redatta da medico specialista che esercita la professione in regime privato, non è possibile ritirare in farmacia direttamente i farmaci in regime di convenzione con il S.S.N;

- è stato eliminato l'obbligo, da parte del medico prescrittore, di conservare per sei mesi le copie delle ricette;

- il farmacista che contravvenga, nella spedizione delle ricette, agli obblighi stabiliti dal testo unico per la vendita di sostanze appartenenti alle prime tre tabelle stupefacenti, salvo il fatto costituisca reato, è soggetto alla sola sanzione amministrativa;

- restano invariati tutti gli altri adempimenti amministrativi relativi all' approvvigionamento, alla custodia in farmacia, all'identificazione di chi ritira il farmaco, alla detenzione e compilazione del registro di carico e scarico (oppioidi in Tabella I, II, III, IV; non contemplato per gli analgesici e altri farmaci contemplati in Tabella V), alla conservazione delle ricette da parte del Farmacista.

\section{MIGLIORARE LA CONTINUITÀ DELL'ASSISTENZA TERRITORIALE E DOMICILIARE}

La nuova normativa cerca anche di inquadrare le possibilità dell' assistenza territoriale e domiciliare, con l'intento di facilitare l'eliminazione della disparità tra il trattamento intra- e extra-ospedaliero. Ecco le principali novità introdotte:

- le unità operative delle strutture sanitarie nonché le unità operative dei servizi territoriali delle Aziende Sanitarie Locali sono dotate di registro di carico e scarico;

- il registro deve essere conforme al modello predisposto dal Ministero della Sanità ed è vidimato dal Direttore Sanitario o suo delegato che provvede alla sua distribuzione e deve essere conservato in ciascuna U.O. dal responsabile dell'assistenza infermieristica per due anni dalla data dell'ultima registrazione;

- il dirigente medico preposto all'U.O. è responsabile della effettiva corrispondenza tra la giacenza contabile e quella reale delle sostanze stupefacenti;

- il direttore responsabile del Servizio Farmaceutico compie periodiche ispezioni per accertare la corretta tenuta dei registri di carico e scarico e redige apposito verbale da trasmettere alla Direzione Sanitaria;

- il paziente in dimissione dal ricovero ospedaliero può ricevere la quantità di medicinale necessaria per continuare la terapia, avendo in questo modo il tempo per procurarsi i medicinali, prescritti con la ricetta autocopiante;

- i farmaci compresi nell' Allegato III-bis possono essere trasportati e consegnati al domicilio del paziente da: personale sani- 
tario che opera nei distretti sanitari, nei servizi territoriali o negli ospedali pubblici o accreditati, da infermieri professionali che effettuano servizi di assistenza domiciliare nell' ambito dei distretti sanitari di base o nei servizi territoriali e da familiari del paziente, opportunamente identificati dal medico o dal farmacista;

- anche i medici sono autorizzati ad approvvigionarsi dei farmaci di cui all'Allegato IIIbis attraverso autoricettazione, su ricetta autocopiante a ricalco;

- i medici sono inoltre autorizzati a detenere, nonché a trasportare, $\mathrm{i}$ farmaci analgesici oppiacei, per uso professionale urgente.

L'obiettivo della continuità assistenziale tra ospedale, territorio e/o domicilio è inoltre alla base dei recentissimi provvedimenti dell' AIFA (Agenzia Italiana del Farmaco), che ha provveduto a riclassificare in fascia A (in qualche caso ricontrattandone il prezzo con le aziende produttrici) alcuni analgesici oppiodi e a approvare nuove formulazioni, collocandole sempre in fascia A, a totale carico del S.S.N. [10]

Le tabelle $1 \mathrm{~A}$ e $1 \mathrm{~B}$ danno conto di queste novità, mentre nelle tabelle $2 \mathrm{~A}$ e $2 \mathrm{~B}$ vengono indicati i costi al pubblico e per il SSN di questi prodotti, nonché l'eventuale sconto obbligatorio fissato per le forniture alle strutture pubbliche del SSN.

L'estensione della durata della validità della ricettazione a trenta giorni solleva una questione interessante, ossia la coerenza delle confezioni con le esigenze di prescrizione. La maggioranza delle confezioni di analgesici oppioidi, sia maggiori che minori, attualmente disponibili in commercio, sono infatti tarate sulla legislazione precedente, come evidenziato nelle tabelle $2 \mathrm{~A}$ e $2 \mathrm{~B}$, ponendo problemi di non banale soluzione al medico che voglia ora prescriverle coerentemente alle nuove possibilità. Fanno eccezione, per quanto riguarda gli oppioidi maggiori, i prodotti a base di ossicodone, che coprono per 28 giorni le esigenze terapeutiche a determinati livelli dose, e alcuni prodotti a base di tramadolo, come le confezioni di Tramadolo Viatris ${ }^{\circledR}$ in capsule da $50 \mathrm{mg}$ e di Tradonal SR $®$, che contengono la quantità di farmaco appropriata per coprire l'intero arco temporale di validità della ricettazione. Si tratta di una questione affatto secondaria, in quanto l'inappropriatezza del confezionamento comporta la necessità di prescrizioni più ravvicinate, con l'indotto del lavoro aggiunto per il medico e delle spese e
Tabella 1A

Provvedimenti dell'AlFA in merito ad alcuni analgesici oppiodi maggiori

\section{Tabella 1B}

Provvedimenti dell'AlFA in merito ad alcuni analgesici oppiodi minori

\begin{tabular}{lcccc}
\multicolumn{1}{c}{$\begin{array}{c}\text { FARMACO } \\
\text { Oppioidi maggiori }\end{array}$} & Brand & $\begin{array}{c}\text { Classe } \\
\text { SSN }\end{array}$ & $\begin{array}{c}\text { Nota } \\
\text { AIFA }\end{array}$ & Provvedimento \\
\hline $\begin{array}{l}\text { Fentanil citrato a rilascio orale } \\
\text { Ossicodone }\end{array}$ & Actiq & A & $/$ & Nuova registrazione \\
$\begin{array}{l}\text { Ossicodone } \mathrm{HCl}+ \\
\text { paracetamolo }\end{array}$ & A & $/$ & Nuova registrazione \\
$\begin{array}{l}\text { Morfina } \mathrm{HCl} \text { a lento rilascio } \\
\text { Depalgos }\end{array}$ & A & $/$ & Nuova registrazione \\
Buprenorfina transdermica & Ticinan & A & $/$ & $\begin{array}{c}\text { Nuova registrazione } \\
\text { Transtec, Triquisic }\end{array}$ \\
\hline
\end{tabular}

\begin{tabular}{ccccc}
\hline $\begin{array}{c}\text { FARMACO } \\
\text { Oppioidi minori }\end{array}$ & Brand & $\begin{array}{c}\text { Classe } \\
\text { SSN }\end{array}$ & $\begin{array}{c}\text { Nota } \\
\text { AIFA }\end{array}$ & Provvedimento \\
\hline Codeina + paracetamolo & Co-Efferalgan & A & 3 & $\begin{array}{c}\text { Variazione regime di } \\
\text { dispensazione }\end{array}$ \\
Tramadolo & $\begin{array}{c}\text { Tradonal, } \\
\text { Tramadolo Viatris, } \\
\text { Fortradol, Contramal, } \\
\text { Prontalgin e altri }\end{array}$ & A & 3 & $\begin{array}{c}\text { Variazione regime di } \\
\text { dispensazione, nuove } \\
\text { registrazioni }\end{array}$ \\
\hline
\end{tabular}




\section{Pradelli}

\begin{tabular}{|c|c|c|c|c|}
\hline $\begin{array}{c}\text { FARMACO } \\
\text { Oppioidi maggiori }\end{array}$ & Confezioni & $\begin{array}{l}\text { Prezzo al } \\
\text { pubblico } \\
\text { (euro) }\end{array}$ & $\begin{array}{l}\text { Prezzo ex } \\
\text { factory } \\
\text { (euro) }\end{array}$ & $\begin{array}{l}\text { Sconto forniture } \\
\text { ospedaliere }\end{array}$ \\
\hline $\begin{array}{l}\text { Fentanil citrato a } \\
\text { rilascio orale }\end{array}$ & $\begin{array}{c}3 \text { pastiglie orosolubili da } \\
200,400,600,800,1200 \text { o } \\
1600 \mathrm{mg}\end{array}$ & 32,18 & 19,5 & $\begin{array}{l}5 \% \text { sul prezzo ex } \\
\text { factory }\end{array}$ \\
\hline \multirow{2}{*}{ Ossicodone } & 28 CPR SR 10 mg & 17,33 & 10,5 & \multirow{2}{*}{$\begin{array}{l}\text { In caso di sforamento } \\
\text { del tetto di spesa } \\
\text { convenzionato }\end{array}$} \\
\hline & 28 CPR SR 20 mg & 34,18 & 20,71 & \\
\hline \multirow{3}{*}{$\begin{array}{l}\text { Ossicodone } \mathrm{HCl}+ \\
\text { paracetamolo }\end{array}$} & $28 \mathrm{CPR} 5 \mathrm{mg}+325 \mathrm{mg}$ & 16,11 & 9,76 & \multirow{3}{*}{$\begin{array}{l}\text { In caso di sforamento } \\
\text { del tetto di spesa } \\
\text { convenzionato }\end{array}$} \\
\hline & 28 CPR $10 \mathrm{mg}+325 \mathrm{mg}$ & 16,11 & 9,76 & \\
\hline & 28 CPR $20 \mathrm{mg}+325$ mg & 16,11 & 9,76 & \\
\hline \multirow{5}{*}{$\begin{array}{l}\text { Morfina } \mathrm{HCl} \text { a lento } \\
\text { rilascio }\end{array}$} & 20 CPR SR 10 mg & 4,65 & 2,82 & \multirow{5}{*}{$\begin{array}{l}\text { In caso di sforamento } \\
\text { del tetto di spesa } \\
\text { convenzionato }\end{array}$} \\
\hline & 20 CPR SR 30 mg & 9,59 & 5,81 & \\
\hline & 20 CPR SR 60 mg & 17,83 & 10,8 & \\
\hline & 20 CPR SR 100 mg & 24,05 & 14,57 & \\
\hline & 20 CPR SR $200 \mathrm{mg}$ & 48,11 & 29,15 & \\
\hline \multirow{3}{*}{$\begin{array}{l}\text { Buprenorfina } \\
\text { transdermica }\end{array}$} & 3 cerotti $20 \mathrm{mg} 35 \mathrm{mcg} / \mathrm{h}$ & 27,38 & 16,59 & \multirow{3}{*}{$\begin{array}{l}15 \% \text { sul prezzo ex } \\
\text { factory }\end{array}$} \\
\hline & 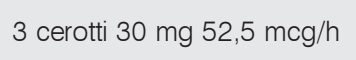 & 40,55 & 24,57 & \\
\hline & 3 cerotti $40 \mathrm{mg} 70 \mathrm{mcg} / \mathrm{h}$ & 50,47 & 30,58 & \\
\hline
\end{tabular}

\section{Tabella 2A}

Prezzi al pubblico e al SSN delle specialità medicinali soggette ai provvedimenti AIFA (oppioidi maggiori) [10]

\begin{tabular}{|c|c|c|c|c|}
\hline $\begin{array}{c}\text { FARMACO } \\
\text { Oppioidi minori }\end{array}$ & Confezioni & $\begin{array}{c}\text { Prezzo al } \\
\text { pubblico } \\
\text { (euro) }\end{array}$ & $\begin{array}{l}\text { Prezzo ex } \\
\text { factory } \\
\text { (euro) }\end{array}$ & $\begin{array}{l}\text { Sconto forniture } \\
\text { ospedaliere }\end{array}$ \\
\hline $\begin{array}{l}\text { Codeina }+ \\
\text { paracetamolo }\end{array}$ & $\begin{array}{c}16 \text { CPR } 30 \text { mg (codeina) + } \\
500 \text { mg (paracetamolo) }\end{array}$ & 4,39 & 2,66 & $\begin{array}{l}\text { In caso di sforamento } \\
\text { del tetto di spesa } \\
\text { convenzionato }\end{array}$ \\
\hline \multicolumn{5}{|l|}{ Tramadolo (alcuni) } \\
\hline \multirow{3}{*}{ Tramadolo VIATRIS } & Gocce $10 \mathrm{ml}$ & 4,62 & 2,8 & \multirow{3}{*}{$\begin{array}{l}\text { In caso di sforamento } \\
\text { del tetto di spesa } \\
\text { convenzionato }\end{array}$} \\
\hline & 30 capsule $50 \mathrm{mg}$ & 6,24 & 3,78 & \\
\hline & 5 fiale da $100 \mathrm{mg}$ & 3,96 & 2,40 & \\
\hline \multirow{4}{*}{ Tradonal SR } & 30 capsule SR $50 \mathrm{mg}$ & 6,35 & 3,85 & \multirow{4}{*}{$\begin{array}{l}\text { In caso di sforamento } \\
\text { del tetto di spesa } \\
\text { convenzionato }\end{array}$} \\
\hline & 30 capsule SR 100 mg & 12,70 & 7,70 & \\
\hline & 30 capsule SR $150 \mathrm{mg}$ & 19,07 & 11,55 & \\
\hline & 30 capsule SR 200 mg & 25,40 & 15,39 & \\
\hline \multirow{8}{*}{$\begin{array}{l}\text { Contramal* e } \\
\text { Fortradol }\end{array}$} & Gocce $10 \mathrm{ml}$ & 4,62 & 2,8 & \multirow{8}{*}{$\begin{array}{l}\text { In caso di sforamento } \\
\text { del tetto di spesa } \\
\text { convenzionato }\end{array}$} \\
\hline & 20 CPS $50 \mathrm{mg}$ & 4,62 & 2,8 & \\
\hline & Gocce $30 \mathrm{ml}^{*}$ & 12,54 & 7,60 & \\
\hline & 20 CPR SR $100 \mathrm{mg}$ & 9,41 & 5,7 & \\
\hline & 10 CPR SR $150 \mathrm{mg}$ & 7,05 & 4,28 & \\
\hline & 10 CPR SR $200 \mathrm{mg}$ & 9,41 & 5,7 & \\
\hline & 5 fiale da $100 \mathrm{mg}$ & 5,78 & 3,50 & \\
\hline & 5 fiale da $50 \mathrm{mg}$ & 3,30 & 2,00 & \\
\hline
\end{tabular}

\section{Tabella 2B}

Prezzi al pubblico e al SSN delle specialità medicinali soggette ai provvedimenti AIFA (oppioidi minori) [10] 


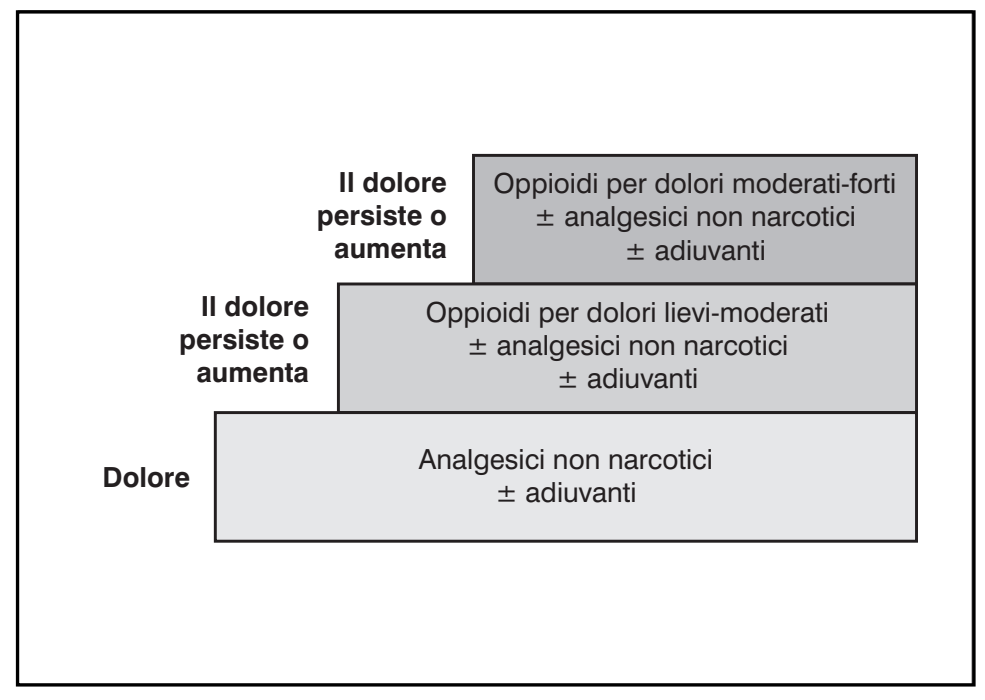

Figura 1

La scala analgesica sequenziale proposta dall'OMS per la gestione del dolore cronico del tempo perso dal paziente in ambulatorio e farmacia.

Un altro punto di novità da notare riguarda i requisiti per la rimborsabilità da parte del SSN di tramadolo e dell' associazione fissa codeina + paracetamolo, soggetta alla nota AIFA (ex note CUF) nr. 3, ossia "limitatamente ai pazienti affetti da dolore lieve o moderato in corso di patologia neoplastica o degenerativa, e sulla base di eventuali disposizioni delle regioni e delle province autonome"[10]. Mentre per codeina + paracetamolo tale indicazione non si discosta da quella registrata ufficialmente in scheda tecnica, per tramadolo essa si configura come una novità.

I prodotti a base di tramadolo commercializzati in Italia, infatti, sono approvati per l'utilizzo nei pazienti con dolore da moderato a severo. Di fatto, dunque, l'AIFA ha considerato che tramadolo, per le sue particolari caratteristiche farmacologiche, possa e debba essere impiegato anche negli stati dolorosi di intensità medio-bassa. Ciò, d'altronde, corrisponde all'utilizzo raccomandato dall' OMS per gli oppioidi deboli (secondo livello della scala a tre gradini, figura 1). Ricordiamo che tramadolo esplica la sua attività analgesica con un meccanismo d'azione legato solo in parte al legame con i recettori per gli oppioidi (da cui anche il bassissimo potenziale di abuso e di dipendenza), svolgendo anche attività inibitoria sulla ricaptazione delle monoamine coinvolte nella trasmissione dello stimolo doloroso.

Mentre in passato si era sottolineato l'approccio progressivo e sequenziale nell'utilizzo dei farmaci dei tre gradini, oggi viene evidenziato come debba essere l'intensità del dolore, e non la sequenzialità dei gradini (né, tantomeno, la prognosi del paziente) a dettare il livello di farmaco con il quale iniziare un trattamento del dolore cronico. Il suggerimento dell' OMS è quello di inserire la strategia terapeutica in un appropriato programma di assistenza continuativa in terapia antalgica e cure palliative. In questo senso, e a prescindere dal livello di discrezionalità nella valutazione dell'intensità del dolore, ancora troppo spesso effettuata con strumenti non standardizzati e validati, appare appropriato il riconoscimento della rimborsabilità del tramadolo ai pazienti con dolore di intensità anche non elevatissima gestiti dalle unità territoriali di terapia del dolore dopo la dimissione dall'ospedale.

L'autorizzazione all'immissione in commercio di nuovi prodotti, ampliando la gamma dei medicinali disponibili, consente inoltre una maggior flessibilità al medico nella scelta dell'oppioide che meglio si adatta alle caratteristiche del paziente, permettendo di tenere in giusta considerazione i molti fattori di variabilità alla risposta a tali farmaci, che dipende da componenti biofarmaceutiche, farmacocinetiche e farmacodinamiche.

\section{CONCLUSIONI}

Nel corso degli ultimi anni, nel nostro Paese si è assistito ad uno sforzo teso ad allineare gli standard nazionali di terapia del dolore a quelli degli altri Paesi dal livello di sviluppo comparabile.

La spinta in questo senso viene sia dagli operatori sanitari coinvolti direttamente nella gestione dei pazienti, i quali da tempo chiedevano misure mirate in queste direzioni, sia dal legislatore, che ha provveduto a delineare un nuovo quadro normativo, gettando le basi per un miglioramento significativo della qualità della terapia antalgica. L'approvazione di queste leggi, tuttavia, costituisce solo un primo passo verso un corretto approccio alla sintomatologia dolorosa.

La divulgazione del contenuto delle leggi e delle linee guida di terapia del dolore nel mondo ospedaliero e sanitario in generale dovrà essere inserita in progetti di ampio respiro, quale quello denominato "Ospedale senza dolore" e la loro conoscenza potrà essere oggetto di corsi di formazione, anche all'interno dei programmi previsti dalle normative sugli hospice. 
In conclusione, negli ultimi anni sono state create le premesse per consentire una gestione razionale e moderna dei pazienti con dolore cronico, cui però debbono ancora far seguito i necessari adattamenti culturali, orga- nizzativi, amministrativi e di buona pratica clinica per conseguire il risultato di garantire un' assistenza efficace ed efficiente alla vastissima popolazione dei pazienti con sintomatologia dolorosa.

\section{BIBLIOGRAFIA}

1. Anon. Achieving Balance in National Opioids Control Policy. Guidelines for Assessement World health Organization. Geneve 2000

2. Minotti V e Betti M. Attitudes of italian generasl practitioners in the treatment of cancer pain. The Committee of the Associazione Italiana di Oncologia Medica (AIOM). Tumori 1997; 83 (4): 729-31

3. Rupp T, Delaney KA. Inadequate analgesia in emergency medicine. Ann Emerg Med. 2004 Apr;43(4):494-503.

4. D.P.R. 309/90 "Testo unico delle leggi in materia di disciplina degli stupefacenti e sostanze psicotrope, prevenzione, cura e riabilitazione dei relativi stati di tossicodipendenza”, G.U./S.O. n. 255, 31-10-1990

5. Joranson DE, Ryan KM, Gilson AM, Dahl JL. Trends in medical use and abuse of opioid analgesics. JAMA. 2000 Apr 5;283(13):1710-4.

6. Legge n. 12, 8/2/2001 “Norme per agevolare l'impiego dei farmaci analgesici oppiacei nella terapia del dolore" G.U.n.41, 19/10/2001

7. Decreto 24/5/2001 “Approvazione del ricettario per la prescrizione dei farmaci di cui all'allegato III bis del D.P.R. 9 ottobre $1990 n^{\circ} 309$ introdotto dalla Legge 8 febbraio $2001 n^{\circ} 12$ ”, G.U. 133, 11/6/2001

8. Decreto 3/8/2001 "Approvazione del registro di carico e scarico delle sostanze stupefacenti e psicotrope per le unità operative” G.U. n ${ }^{\circ} 204,3 / 9 / 2001$

9. Decreto 4/4/2003 “Modifiche ed integrazioni al Decreto del Ministro della Sanità del 24 maggio 2001 ” G.U. $\mathrm{n}^{\circ} 122,28 / 5 / 2003$

10. Comunicati di Registrazione Nuovi Prodotti Analgesici e Determinazioni AIFA sulla Rimborsabilità, G.U.n ${ }^{\circ} 1,3 / 1 / 2005$ 